\title{
Metacognitive and online error awareness deficits after prefrontal cortex lesions.
}

\author{
Hoerold D, Pender NP, Robertson IH.
}

\begin{abstract}
Awareness of deficits after brain injury represents a significant clinical and theoretical challenge, but relatively little is known about the neuroanatomical correlates of specific types of deficit awareness. We examined the awareness correlates of left versus right prefrontal cortex lesions in comparison to left and right posterior lesions including two types of awareness measures--metacognitive and online error monitoring. Frontal lobe frontal lesion patients exhibited impaired metacognitive awareness and also showed deficits in monitoring errors as they occurred. In addition, frontal lobe lesion patients also showed reduced autonomic response to aware errors. Online and metacognitive awareness were not, however, significantly correlated, suggesting that distinct neuroanatomical systems may underpin these two types of awareness deficit. We hypothesize that while metacognitive awareness depends on both left and right frontal regions, accurate moment-to-moment processing of errors depends more on the right than on the left prefrontal cortex.
\end{abstract}

Keywords

Deficit awareness; Insight; Error monitoring; Error awareness; Brain lesions

\section{Introduction}

Impaired awareness of deficit resulting from neural injury represent a substantial clinical problem, with numerous studies documenting the negative impact of insight on family members and caregivers (e.g., Prigatano, Borgaro, Baker, \& Wethe, 2005), but also on rehabilitation effectiveness, vocational outcome (e.g., Prigatano, 2005) and treatment compliance (Worrall, Chen, Dimberg, \& Katz, 2005). Over the past 20 years, such impaired deficit awareness has increasingly been recognised as a multifaceted neuropsychological problem, as distinct from a unitary psychiatric condition (Schacter \& Prigatano, 1991), and theoretical models have since been proposed which distinguish between different types of deficit awareness. The most common classification differentiates between 'metacognitive' or 'intellectual' awareness, as separate from 'online' awareness (e.g. Crosson et al., 1989; Toglia \& Kirk, 2000). These models view metacognitive awareness as an overall knowledge of one's disorder, while online awareness includes both the ability to recognize errors as they occur (online emergent awareness) and the ability to anticipate problems before they occur (online anticipatory awareness) (Toglia \& Kirk, 2000). Whether these aspects of awareness interact in a hierarchical manner is currently under debate (e.g., Abreu et al., 2001; Abu-Akel \& Shamay-Tsoory, 2011), with some

\footnotetext{
- Corresponding author. Present address: Institute of Psychiatry, King's College London, Windsor Walk, Denmark Hill, London, SE5 8AF, United Kingdom.

Tel.: +44 207848 0733; fax: + 442078480260.

E-mail address: doreen.hoeroldøkcl.ac.uk (D. Hoerold).
}

findings suggesting that the two online components of selfawareness may be closely related to each other, but not necessarily to metacognitive awareness (O'Keeffe, Dockree, Moloney, Carton, \& Robertson, 2007a). This is important, since most studies typically assess deficit-awareness using questionnaire discrepancies between patients' self-report and that of a significant other (Fleming, Strong. \& Ashton, 1996), and thus arguably only measure metacognitive awareness, while neglecting other, more subtle aspects of awareness.

While the study of impaired deficit awareness among patients with traumatic brain injury (TBI) and degenerative disease such as Alzheimer's disease and fronto-temporal dementia has flourished over recent years, it must be acknowledged that these patients suffer from widespread neural damage, and hence even moderately precise brain-behaviour relationships are difficult to establish. What these patient groups have in common, however, is their vulnerability to frontal systems dysfunction, and indeed, many neuroanatomical models have emphasized the role of fronto-striatal circuits for higher cognitive functions including conscious self-awareness (e.g., Stuss, 1991; Stuss, Picton, \& Alexander, 2001).

Theoretical models distinguishing between online awareness of errors and metacognitive knowledge may also be useful for the study of impaired awareness in specific neurological syndromes such as anosognosia following stroke. Within this population, it has been suggested that anosognosia may be the result of a failure of motor monitoring (e.g., Jenkinson, Edelstyn, Drakeford, \& Ellis, 2009), which fits well with the notion of impaired error awareness potentially leading to difficulties in metacognitive understanding that one has an illness. Neuroanatomically, a number of 
studies have suggested that there may be asymmetry in deficit awareness among anosognosia patients, with right hemisphere, and in particular right frontal impairment, beings associated with greater deficit awareness. For example anosognosia for hemiparesis has been found to be particularly associated with right fronto-parietal dysfunction (see Pia, Neppi-Modona, Ricci, \& Berti, 2004: Orfei et al., 2007 for reviews). Similarly, Vocat, Staub, Stroppini, and Vuilleumier (2010) report that networks involving right premotor, cingulate gyrus, temporoparietal junction and medial temporal lobe areas were implicated in chronic anosognosia for hemiplegia. In Alzheimer's disease, a number of studies have used single photon emission computed tomography (SPECT) to show that hypoperfusion of right dorsolateral prefrontal cortex us associated with patients' deficit awareness deficits (e.g., Starkstein et al., 1995).

No study has hitherto examined metacognitive and online awareness deficits in a focal lesion population. Thus, following from our previous research on these separate deficit awareness subtypes in traumatic brain injury (O'Keeffe et al., 2007a) and dementias known to involve atrophy of the frontal lobes (O'Keeffe et al. 2007b), in this study we test the hypothesis that frontal lobe lesions will be associated with one or both of these types of awareness deficit. Previous work from our lab has shown that impaired deficit awareness may be mediated by poor processing of errors associated with lowered autonomic response to errors as shown by skin conductance responses (SCR) (O'Keeffe, Dockree, \& Robertson, 2004) and so we had an additional interest in testing a second hypothesis, that these responses will also be diminished in right frontal lesions patients. As the frontal lobe model of awareness was of particular relevance here, we focussed on recruitment of those patients with predominantly unilateral brain lesions in the frontal lobe or outside the frontal lobe, without further neuroanatomical specificity for non-frontal regions.

\section{Method}

\subsection{Participants}

Patients for this study were recruited on a voluntary basis through the Department of Psychology in Beaumont Hospital. Dublin, using the following inclusion criteria: Patients must be between the ages of 18 and 70 years, and have received a diagnosis of a focal brain lesion in any of the four cortical regions specified - right or diagnosis of a focal brain lesion in any of the four cortical regions specified-right or lesions did not include the motor cortex. Patients were also excluded from the study if any of the following applied: a history of alcohol/substance abuse, diagnosis of a major psychological/psychiatric disorder, any additional neurological disorder, current disorientation, aphasic difficulties, visuospatial impairment or neglect. This led to a sample of 12 left frontal (LF) and 7 right frontal (RF), as well as 14 left nonfrontal (LNF) and 12 right non-frontal (RNF) focal lesion patients (total=45). Patient groups did not differ significantly for time since lesion (TSL) $[F(3,41)=2.03 ; p>0.05]$. Table 1 displays the clinical details for each patient, including diagnosis, time since lesion (TSL) and the type of clinical brain scan available. MRI scans were available for 29 patients, while CT images were provided for 16 patients. Representative slices of patients MRI or CT scans are shown in Fig. 1a-d. Since all scans were clinical in nature and thus varied considerably in orientation and other parameters, lesions are shown on an individual basis, rather than on reconstructed lesion maps.

A sample of 15 healthy volunteer participants served as a control group. Exclusion criteria were the same as for patients, with the additional requirement that control participants must never have suffered a head injury leading to a loss of consciousness or memory.

Ethical approval for the study was obtained from the research ethics committees of Beaumont Hospital and Trinity College Dublin. Informed consent was obtained from all participants according to the Declaration of Helsinki. All participants received reimbursement for travel expenses.

\section{Materials and procedure}

All participants completed a testing session of approximately $2.5 \mathrm{~h}$, which included the following neuropsychological measures:
2.2.1. Screening measures

Revised National Adult Reading Test for premorbid IQ estimate (NART-R; Nelson, 1982).

Hospital Anxiety and Depression Scale (HADS: Zigmond \& Snaith. 1983) for symptoms of anxiety and depression.

\subsubsection{Neuropsychological measures}

\subsubsection{Memory}

2.2.2.1.1. Working memory. Digit span and Spatial span subtests from the Wechsler Memory Scale (WMS) III (Wechsler, Wycherly, Benjamin, Crawford, \& Mockler, 1998)

2.2.2.1.2. Verbal memory. Logical memory (immediate and delayed) subtest from the WMS III

2.2.2.1.3. Visual memory. Faces (immediate and delayed) subtest from the WMS III.

All test scores were converted into scaled scores, which have a mean of 10 and a standard deviation of 3.

\subsubsection{Attention}

2.2.2.2. Visuomotor attention. Trail making test (TMT; Partington \& Leiter, 1949) - total number of errors made in part B.

2.2.2.2. Sustained attention. Participants were required to perform three blocks of the fixed-sequence version of the sustained attention to response task (SART; Manly et al., 2003; Robertson, Manly, Andrade, Baddeley, \& Yiend, 1997) Participants were instructed to press the left mouse button for all numbers presented in fixed ascending sequence, and withhold responses for the number ' 3 '. The error score is the percentage of commission errors across all blocks (commission errors/ number of no-go targets $\times 100$ ).

\subsubsection{Executive functioning}

2.2.2.3.1. Verbal fluency. For letters F, A and S, the total number of items generated during 60 s served as the score.

2.2.2.3. Semantic fluency. The total number of animals named within $60 \mathrm{~s}$ (Spreen \& Strauss, 1998).

\subsubsection{Awareness measures}

2.2.3.1. Metacognitive awareness Three questionnaires were administered to investigate metacognitive awareness:

Frontal Systems Behavior Scale (FrSBe-Other) (Grace \& Malloy, 2002), which rates the frequency of symptoms associated with frontal systems damage. Higher scores indicate more symptomatology.

Cognitive Failures Questionnaire (CFQ-Other) (Broadbent, Cooper, FitzGerald, \& Parkes, 1982), which rates the frequency of everyday cognitive failures and absentmindedness. Higher scores indicate more symptomatology.

Patient Competency Rating Scale (PCRS-Other) (Prigatano \& Fordyce, 1986), which rates participants' competencies on activities of daily living. interpersonal, cognitive and emotional competencies. Higher scores indicate higher competencies on the PCRS.

We measured metacognitive awareness by calculating discrepancies between self- and other ratings on the CFQ. FrSBe and PCRS ('Self minus 'Other' for CFQ and FrSBe; 'Other' minus 'Self for PCRS'), so that negative discrepancy scores indicate an under-estimation of difficulties. A compound z-score served as the metacognitive awareness score.

2.2.3.2. Online emergent awareness

2.2.32.1. Error awareness on SART. Participants were required to verbally indicate awareness of commission errors on the SART as they occurred ("Hit!") All digits were presented for $300 \mathrm{~ms}$ and $800 \mathrm{~ms}$ inter-stimulus interval, in a fixed and repeating sequences from 1 to 9 .

2.2.32.2. Skin conductance response ( $S C R$ ) during $S A R T$. SCR was measured during SART performance, using a Biopac Systems Inc. MP30 unit, electrodes an software (www.biopaccom). Two Ag/AgCl electrodes, mounted in individual housings and shielded to minimize noise interference, were filled with $\mathrm{SIGMA}^{\mathrm{R}}$ gel and attached to the distal phalanges of the index and middle fingers of participants

${ }^{1}$ Since the PCRS measures competency rather than rather than symptoms, higher scores reflect higher competency. Therefore, when computing discrepancies, we calculated "other" scores minus "self" scores, so that negative discrepancy scores on the PCRS would indicate unawareness, consistent with the other questionnaires used in this study. 
Table 1

Clinical data for focal lesion patients.

\begin{tabular}{|c|c|c|c|}
\hline Patient ID & Diagnosis & TSL (months) & Neuro-imaging \\
\hline & LF group $(N=12)$ & & \\
\hline $008 \mathrm{OC}$ & Grade III glioma & 65 & CT \\
\hline $016 \mathrm{MF}$ & Anaplastic Glioma & 36 & MRI \\
\hline $047 \mathrm{PC}$ & Low grade glioma & 29 & MRI \\
\hline $115 \mathrm{BD}$ & Contusion $(\mathrm{HI})$ & 91 & CT \\
\hline $239 \mathrm{BM}$ & Lacunar infarct & 86 & $\mathrm{CT}$ \\
\hline $291 \mathrm{JOR}$ & High grade glioma (resected) & 144 & MRI \\
\hline $348 \mathrm{RS}$ & Grade Il glioma & 47 & MRI \\
\hline 352 ов & Grade Il glioma & 31 & MRI \\
\hline 355 DJ & Grade Il glioma & 15 & MRI \\
\hline $356 \mathrm{JD}$ & Grade III glioma & 15 & MRI \\
\hline $360 \mathrm{JOR}$ & Grade 1 meningioma & 17 & MRI \\
\hline \multirow[t]{2}{*}{$363 \mathrm{BMG}$} & ACA aneurysm (coiled) & 23 & MRI \\
\hline & RF group $(N=7)$ & & \\
\hline $021 \mathrm{PH}$ & Medial FL. aneurysm (clipped) & 30 & $\mathrm{CT}$ \\
\hline $090 \mathrm{BBD}$ & High grade glioma & 72 & $\mathrm{CT}$ \\
\hline $164 \mathrm{MH}$ & AVM (embolization) & 79 & MRI \\
\hline $335 \mathrm{JB}$ & Grade Il glioma & 24 & MRI \\
\hline $350 \mathrm{CH}$ & Meningioma with associated haemorrhage & 26 & MRI \\
\hline 353 SK & Grade IV glioma & 11 & MRI \\
\hline \multirow[t]{2}{*}{$361 \mathrm{KG}$} & Grade III glioma (debulked) & 12 & MRI \\
\hline & LNF group $(N=14)$ & & \\
\hline $002 \mathrm{CFB}$ & Parieto-occipital AVM & 94 & MRI \\
\hline $049 \mathrm{GC}$ & Temporal lobe meningioma & 43 & MRI \\
\hline $161 \mathrm{CG}$ & PCA aneurysm & 99 & $\mathrm{CT}$ \\
\hline $166 \mathrm{JC}$ & Temporo-occipital subdural haematoma & 74 & $\mathrm{CT}$ \\
\hline $259 \mathrm{SM}$ & Parietal cavernous angioma and intracranial haematoma & 154 & $\mathrm{CT}$ \\
\hline $266 \mathrm{VM}$ & MCA aneurysm (clipped) & 147 & CT \\
\hline $328 \mathrm{SW}$ & Temporo-parietal low grade glioma & 148 & MRI \\
\hline $334 \mathrm{MU}$ & Focal cortical atrophy in posterior parietal/occipital lobe & 144 & MRI \\
\hline $336 \mathrm{PC}$ & Occipital haemorrhage due to suspected AVM & 17 & CT \\
\hline $338 \mathrm{GC}$ & Mesial temporal low grade glioma & 34 & MRI \\
\hline $339 \mathrm{MF}$ & Mesial temporal sclerosis (hippocampectomy) & 55 & MRI \\
\hline 344 SR & Parietal low grade glioma & 29 & MRI \\
\hline $345 \mathrm{AP}^{\mathrm{P}}$ & Parieto-occipital AVM with associated haemorrhage & 11 & MRI \\
\hline \multirow[t]{2}{*}{$347 \mathrm{AA}$} & PCA aneurysm (coiled) & 20 & $\mathrm{CT}$ \\
\hline & RNF group $(N=12)$ & & \\
\hline $046 \mathrm{AB}$ & MCA territory non-haemorrhagic infarction & 20 & $\mathrm{CT}$ \\
\hline $066 \mathrm{GON}$ & Inferior temporal extradural haematoma & 20 & $\mathrm{CT}$ \\
\hline $086 \mathrm{CB}$ & Occipito-parietal haemorrhage and haematoma & 113 & MRI \\
\hline $162 \mathrm{PH}$ & PCA aneurysm (clipped) & 118 & CT \\
\hline $337 \mathrm{CC}$ & Temporal lobe AVM (excised) & 10 & $\mathrm{CT}$ \\
\hline $341 \mathrm{GC}$ & Cavernous haemangioma (resected) & 66 & $\mathrm{CT}$ \\
\hline $342 \mathrm{MK}$ & Hippocampal sclerosis (anterior hippocampectomy) & 31 & MRI \\
\hline $343 \mathrm{GF}$ & Hippocampal sclerosis (anterior hippocampectomy) & 28 & MRI \\
\hline $349 \mathrm{MON}$ & Mesial temporal sclerosis & 36 & MRI \\
\hline 351 EM & Mesial temporal sclerosis (hippocampectomy) & 30 & MRI \\
\hline $357 \mathrm{EQ}$ & Mesial temporal sclerosis (amygdalo-hippocampectomy) & 16 & MRI \\
\hline $358 \mathrm{~EB}$ & Temporal grade II meningioma & 13 & MRI \\
\hline
\end{tabular}

Abbreviations: $\mathrm{ACA}=$ anterior cerebral artery: $\mathrm{AVM}=$ anteriovenous malformation; $\mathrm{CT}=$ computerized tomography; $\mathrm{HI}=$ head injury: $\mathrm{LF}=$ left frontal; $\mathrm{LNF}=$ left non-frontal; $\mathrm{MCA}=$ middle cerebral artery: $\mathrm{MRI}=$ magnetic resonance imaging (structural); $\mathrm{PCA}=$ posterior communicating artery; $\mathrm{RF}=$ right frontal; RNF=right non-frontal; TSL=time since lesion (in months).

non-dominant hand. SCR signals were converted to digital signals via the MP30 unit and processed with BIOPAC Student Lab PRO for offline analysis. For SCR amplitude measurements, a latency window of $1-5 \mathrm{~s}$ following presentation of no-go targets (digit ' '3") was specified. SCR amplitudes ( $\mu$ S) reflect conductance at the peak minus conductance prior to the response, as defined by Stern, Ray, and Quigley (2001). The criterion for the smallest scorable SCR was set at $0.02 \mu \mathrm{S}$. SCR amplitudes were averaged and analyzed for emotionally non-significant stimuli (correct withholds for digit ' 3 ") and aware errors. We have previously found that the fixed-sequence SART lends itself well to the measurement of SCR to error responses, due to its relatively slow presentation and predicable sequence (see O'Keeffe et al., 2004).

\section{Results}

\subsection{Demographic and screening results}

Demographic, screening and neuropsychological data for each patient group and controls are presented in Table 2. Groups did not differ on age $[F(4,60)=0.51 ; p>0.05]$, or years of education $[F(4,60)=1.74, p>0.05]$. One group difference emerged for premorbid $I Q[F(4,60)=4.39, p<0.01]$, with Bonferroni comparisons showing that RNF lesion patients had lower premorbid IQ scores than controls $(p<0.05)$. As a result, all subsequent group comparisons were conducted while co-varying for premorbid IQ scores. No group difference existed for anxiety or depression scores (both $p>0.05$ ).

\subsection{Neurocognitive performance}

Separate univariate ANOVAs, followed by Bonferroni comparisons where indicated, showed that group differences existed on a number of neuropsychological measures. This is also detailed in Table 2, which shows mean neuropsychological performance scores for all groups. 
a

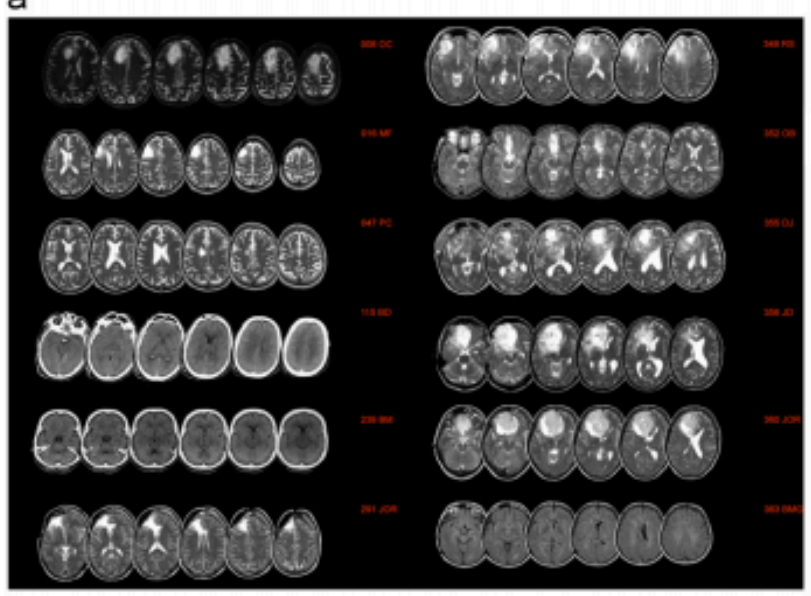

C

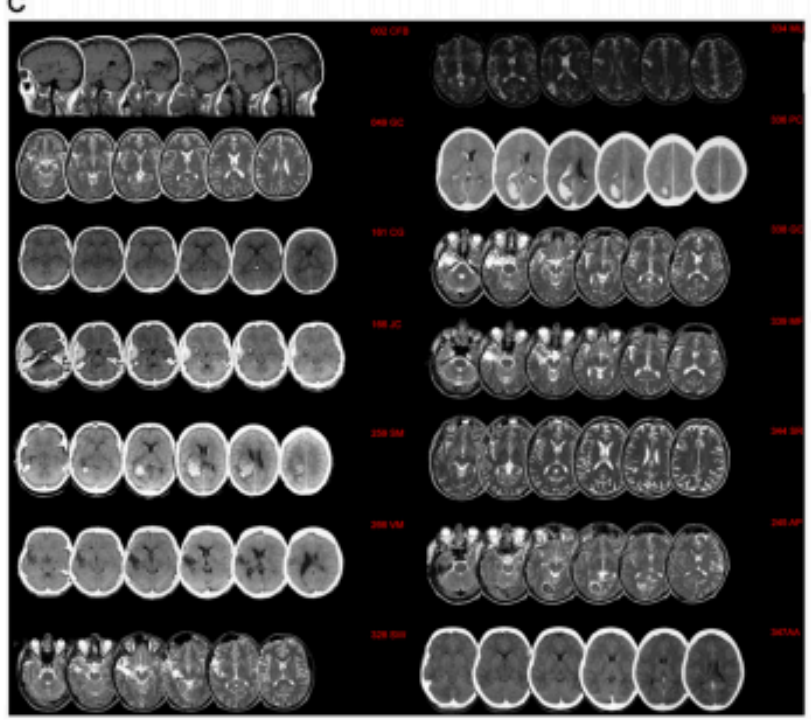

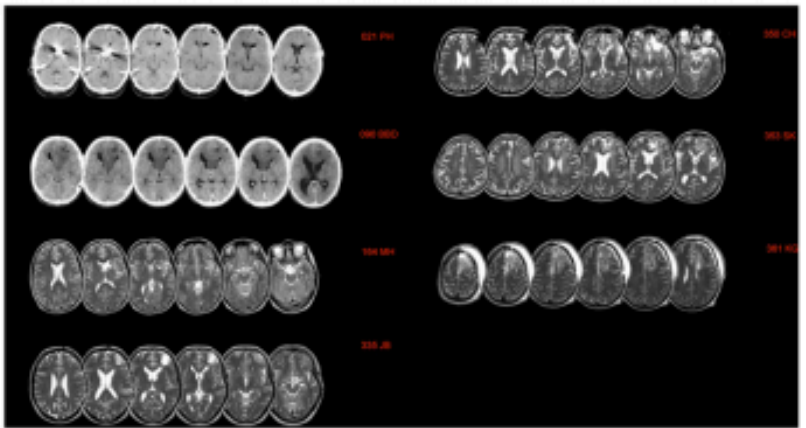

d

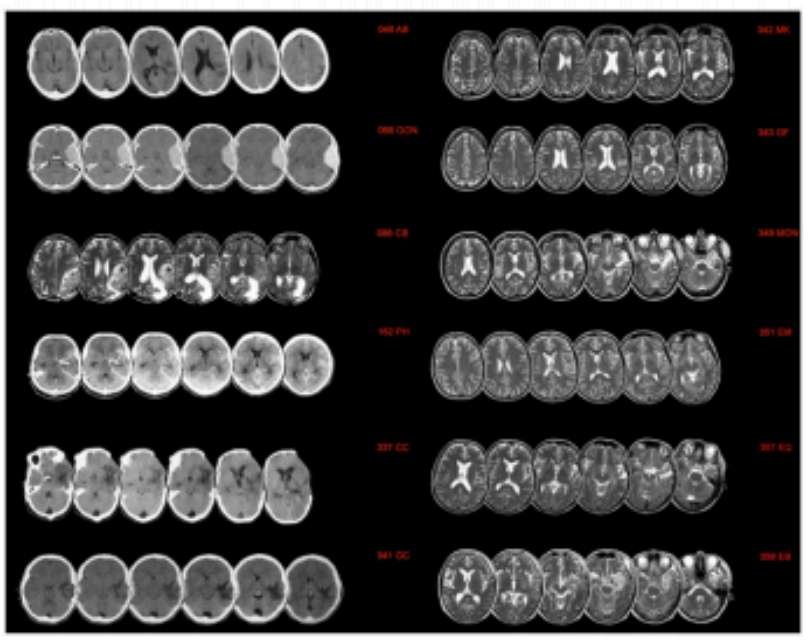

Fig. 1. (a) CT and MRI data for LF lesion patients. (b) CT and MRI data for RF lesion patients. (c) CT and MRI data for LNF lesion patients. (d) CT and MRI data for RNF lesion patients.

For working memory measures, LF lesion patients differed significantly from controls on digit span $[F(4,57)=4.29 ; p<0.01]$ and a main effect of group also approached significance for spatial span ( $p=0.055)$, with mean values suggesting the same pattern of LF lesion patients performing poorer than controls. With regard to verbal memory, on immediate recall of the logical memory task, a group effect also emerged, whereby LF lesion patients again showed significantly lower scores than Controls $[F(4,50)=2.58$; $p<0.05]$. No group difference existed for delayed recall of logical memory. On visual memory measures, groups differed significantly on immediate recognition of WMS faces $[F(4,55)=3.16$; $p<0.05]$, with Bonferroni comparisons revealing that LNF patients scored significantly lower than controls. Groups also differed on delayed recognition of WMS faces $[F(4,55)=3.76 ; p<0.05]$, with Bonferroni comparisons confirming that RNF lesion patients scored significantly lower than controls.

For visuomotor attention, an effect of group approached statistical significance for TMT error scores $(p=0.056]$, and Bonferroni comparisons showed that LF patients differed significantly from controls, from LNF and from RNF patients (all $p<0.05$ ).
Inspection of mean values suggests that both LF and RF lesion groups made more errors than controls or non-frontal lesion groups. On the sustained attention task (SART), LF lesion patients made significantly more errors than controls and RNF patients $\left[F^{\prime}(4,60)=6.47, p<0.01\right]$.

On measures of executive functioning, groups differed significantly on verbal fluency $[F(4,60)=6.92, p<0.01]$. Bonferroni comparisons highlighted that controls performed higher than LF, LNF and RNF lesion patients (all $p<0.05$ ). LF patients also differed significantly from RF lesion patients $(p<0.05$ ). Lastly, on semantic fluency, groups differed again $[F(4,60)=2.60, p<0.05]$, and Bonferroni comparisons confirmed that LF lesion patients performed significantly poorer than controls $(p<0.05)$.

\subsection{Impairment on everyday tasks}

To assess group differences in impairment more broadly, we examined 'Other' ratings on the FrSBe, CFQ and PCRS. Bonferroni comparisons showed that groups did not differ significantly 
Table 2

Demographic and neurocognitive data for all groups.

\begin{tabular}{|c|c|c|c|c|c|c|}
\hline & L.F & RF & LNF & RNF & c & $p$-Value \\
\hline \multicolumn{7}{|l|}{ Demographics and screening } \\
\hline$N(\mathrm{~m}, \mathrm{f})$ & $12(6,6)$ & $7(3,4)$ & $14(7,7)$ & $12(6,6)$ & $15(10,5)$ & - \\
\hline Age (SD) & $46.6(10.1)$ & $40.1(12.5)$ & 42.7 (10.5) & $40.4(14.6)$ & $42.6(12.7)$ & 0.731 \\
\hline Education (years) (SD) & $13.5(4.1)$ & $15.1(2.2)$ & $16.2(3.9)$ & $14.7(3.5)$ & $16.3(1.7)$ & 0.154 \\
\hline Premorbid IQ (SD) & $111.6(8.5)$ & $118.0(7.0)$ & $113.6(8.2)$ & $106.7(14.4)^{4}$ & $119.4(4.2)^{4}$ & 0.004 \\
\hline HADS anxiety (SD) & $6.5(3.7)$ & $6.7(3.6)$ & $7.1(4.6)$ & $6.3(3.3)$ & $5.5(2.2)$ & 0.804 \\
\hline HADS depression (SD) & $3.2(2.7)$ & $2.0(2.2)$ & $4.6(3.8)$ & $2.9(2.4)$ & $2.7(2.0)$ & 0.277 \\
\hline \multicolumn{7}{|l|}{ Memory } \\
\hline Digit span (SD) & $8.3(2.9)^{b}$ & $12.5(3.1)$ & $10.9(3.5)$ & $10.2(2.7)$ & $12.7(3.1)^{\mathrm{b}}$ & 0.005 \\
\hline Spatial span (SD) & $7.4(2.9)$ & $10.5(2.1)$ & $10.1(3.9)$ & $8.4(3.7)$ & $10.9(2.8)$ & 0.055 \\
\hline \multicolumn{7}{|l|}{ Verbal memory: } \\
\hline Logical memory -immediate (SD) & $7.9(3.7)^{\mathrm{b}}$ & $10.4(2.2)$ & $9.4(4.3)$ & $10.4(2.4)$ & $11.8(2.8)^{\mathrm{b}}$ & 0.047 \\
\hline Logical memory delayed (SD) & $8.9(4.3)$ & $10.1(2.7)$ & $10.5(4.4)$ & $10.3(3.3)$ & $12.3(2.8)$ & 0.199 \\
\hline \multicolumn{7}{|l|}{ Visual memory: } \\
\hline Faces immediate (SD) & $10.0(3.7)$ & $10.4(2.4)$ & $9.5(2.8)^{c}$ & $9.3(2.8)$ & $12.7(2.4)^{\mathrm{c}}$ & 0.021 \\
\hline Faces delayed (SD) & $10.1(3.6)$ & $10.0(1.9)$ & $10.2(3.2)$ & $9.1(2.4)^{2}$ & $12.9(2.1)^{2}$ & 0.010 \\
\hline \multicolumn{7}{|l|}{ Attention } \\
\hline TMT errors (SD) & $0.9(0.9)$ & $1.0(1.1)$ & $0.5(0.8)$ & $0.2(0.4)$ & $0.2(0.4)$ & 0.056 \\
\hline \multicolumn{7}{|l|}{ Sustained attention: } \\
\hline SARI error rate $\mathscr{Z}(\mathrm{SD})$ & $19.3(11.2)^{\text {hed }}$ & $9.3(6.3)$ & $12.2(7.7)$ & $9.5(5.3)^{d t}$ & $5.93(4.0)^{b}$ & 0.000 \\
\hline \multicolumn{6}{|l|}{ Executive functioning } & 0.000 \\
\hline Semantic fluency (SD) & $17.5(8.7)^{\mathrm{b}}$ & $19.4(5.0)$ & $20.9(6.4)$ & $21.4(6.7)$ & $25.1(4.1)^{\mathrm{b}}$ & 0.046 \\
\hline
\end{tabular}

Abbreviations: $\mathrm{C}=$ control group; HADS=Hospital Anxiety and Depression Scale; LF=left frontal lesion; LNF= left non-frontal lesion; RF=right frontal lesion; RNF=right non-frontal lesion; SART = sustained attention to response task: SD =standard deviation; TMT = trail making test.

Post-hoc comparisons (Bonferroni-corrected):

${ }^{2}$ RNF vs. $C(p<0.05)$.

LF vs. C $(p<0.05)$.

L.NF v5. C $(p<0.05)$.

A $2 N E$ V $v S, C(p<0.05)$

L.F vs. RF $(p<0.05)$.

(all $p>0.05$ ), although the difference between Controls and LF patients approached significance on all three questionnaires.

\subsection{Metacognitive awareness}

Discrepancy scores were derived by calculating differences between 'Self' and 'Other' ratings on three questionnaires, the CFQ PCRS and FrSBe. Discrepancies were converted into individual $z$-scores and subsequently combined into a compound $z$-score for metacognitive awareness. A univariate ANOVA showed a main effect of group $[F(4,60)=4.94, p<0.01]$. Bonferroni comparisons detected significant differences between LF lesion patients and Controls, LNF and RNF patients (all $p<0.05$ ). Mean $z$-score values for both $\mathrm{LF}$ and RF patients were negative, suggesting that both frontal lesion groups under-estimated their difficulties. In contrast, both non-frontal patient groups, as well as controls, had a tendency to over-estimate their difficulties. Fig. 2 shows $z$-scores for all groups.

\subsection{Online emergent awareness}

\subsubsection{Error awareness during SART}

SART error awareness was calculated as the percentage of all commission errors indicated as aware during the SART. One RNF patient who made no commission errors was excluded from the analysis, leaving only participants with more than one commission error. A univariate ANOVA showed a main effect of group for awareness $[F(4,59)=5.18, p<0.01]$. Bonferroni comparisons indicated that RF, LF and LNF lesion patients showed reduced error awareness, compared to controls $(p<0.05)$. RF lesion patients showed lowest error awareness.

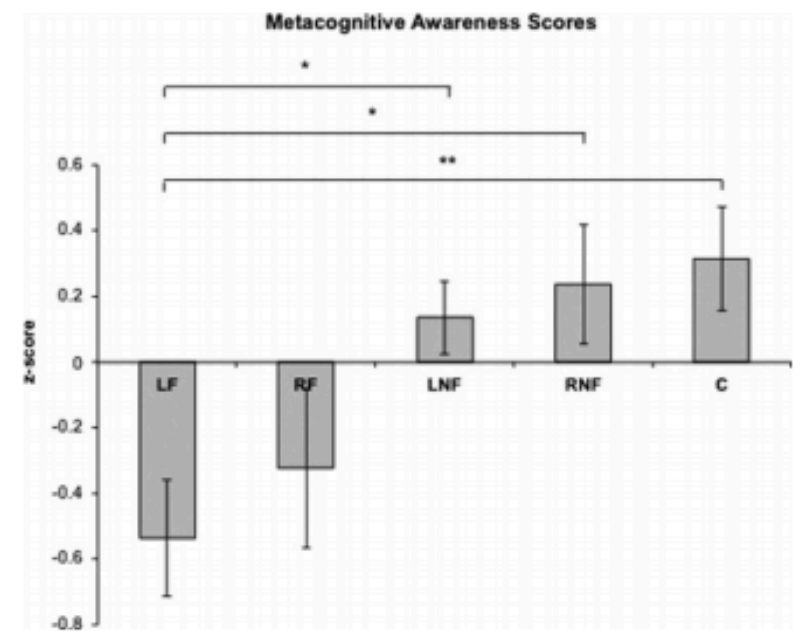

Fig. 2. Metacognitive awareness.

3.5.2. Skin conductance response to aware errors

To examine general autonomic reactivity among all participants, SCR amplitudes to correct withholds were averaged for each participant. A univariate ANOVA showed that groups did not differ in SCR to withhold responses. SCR amplitudes for errors reported as aware were then averaged for each participant. Since not all participants made aware errors, this analysis was performed on a reduced sample as follows: $L F N=9$, RF $N=4, L N F=12$, RNF $N=10$, controls $N=13$. To account for this, 
A

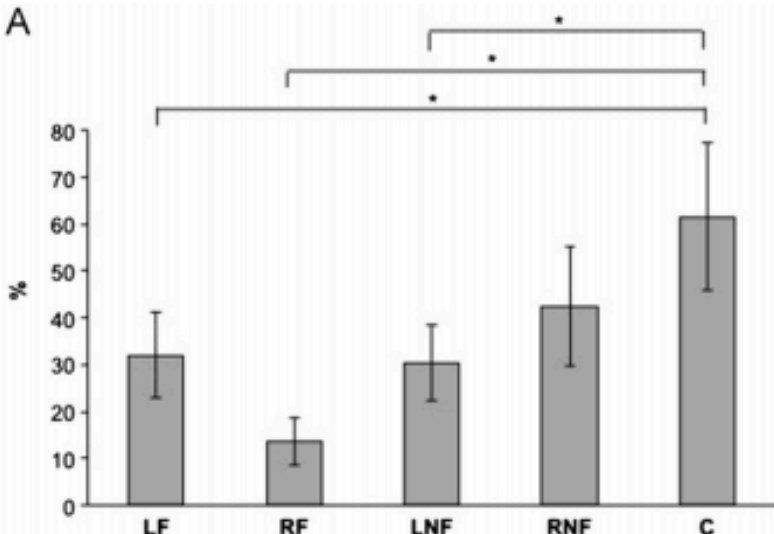

B

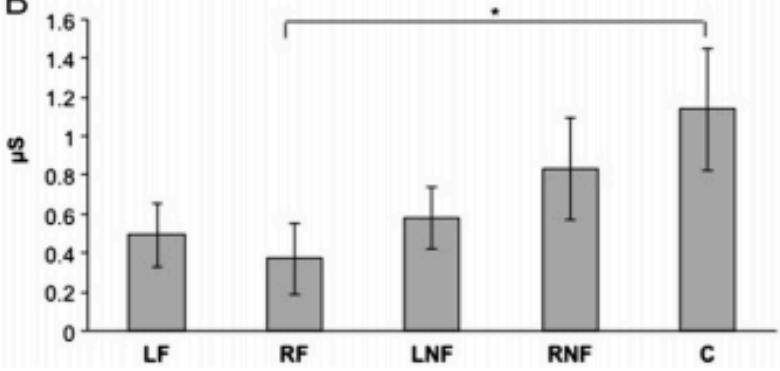

Fig. 3. Online emergent awareness.

non-parametric tests were performed, with a Kruskal-Wallis test highlighting the presence of significant differences between groups $(p<0.05)$. Tamhane post-hoc pair-wise comparisons indicated that RF lesion patients had significantly reduced SCR amplitudes compared to controls $(p<0.05)$. Fig. 3 displays error awareness and SCR amplitudes for all groups.

\subsection{Relationships between awareness types and time since lesion}

In order to examine potential relationships between online emergent awareness, SCR response to aware errors, metacognitive awareness and time since lesion, partial correlations were performed across all patients ( $N=47$ ), controlling for the effect of group and NART IQ. Time Since Lesion did not correlate with either aspect of Metacognitive or Online Emergent Awareness (all $p>0.05$ ). A small positive correlation between SCR response to aware errors and metacognitive awareness approached statistical significance ( $r=0.28, p=0.059$ ), however, this is not a robust effect once appropriate Bonferroni corrections are applied.

\section{Discussion}

The current study aimed to shed light on the putative neuroanatomical bases of deficit awareness by assessing the effects of focal cortical lesions on a number of measures deficit awareness. Specifically, we examined performance of unilateral prefrontal lesion patients, as well as patients with unilateral lesions outside the frontal lobe, on metacognitive awareness and online emergent error awareness.

We initially examined neurocognitive performance among all participants. This confirmed that controls generally performed better than lesion patients, and also suggested that this difference was most pronounced between controls and left frontal patients, across a range of tasks. We also assessed whether groups differed in terms of impairment on everyday tasks, as captured by informant ratings on three questionnaires. This did not seem to be the case: Questionnaire ratings showed that predictably, all patients scored higher on impairment than controls, lesion groups did not differ from one another on impairment. Questionnaire discrepancy data between 'Self' and 'Other' ratings then showed that both left and right frontal lesion patients under-estimated their difficulties, and thus can be considered to exhibit impaired metacognitive awareness. Additionally, we measured online error awareness, by asking patients to monitor their performance during a simple task of sustained attention, and verbally indicate their error awareness. Although all patients showed lower awareness than controls, this was most pronounced among RF lesion patients. When examining only aware errors, both RF and LF lesion patients also showed a decreased autonomic skin conductance response (SCR), again pronounced in RF patients.

We propose that one possible mechanism accounting for deficit awareness impairments following frontal lobe damage may be a deficit in error monitoring. mediated by lowered autonomic arousal, for which prefrontal cortex functioning is crucial. Neuroimaging results such as those of Beauregard, Levesque, and Bourgouin (2001) have shown that right prefrontal cortex plays a prominent role in the regulation of autonomic nervous system function. More specifically, Zahn, Grafman, and Tranel (1999) have demonstrated that right prefrontal cortex lesions impair the generation of SCRs to psychologically significant stimuli, rather than impairing SCRs per se. This was confirmed by our own observation that SCRs to more neutral stimuli, such as correctly withheld responses to the digit 3 , were not impaired in our patient groups. Our group's previous findings have shown that in TBI patients, a specific deficit exists in generating an arousal response to errors, as indexed by lowered SCR amplitudes, which suggests that a link exists between rightlateralized autonomic arousal system functioning and error awareness (O'Keeffe et al., 2004).

Many authors have noted that unawareness of hemiplegia, following right fronto-parietal injury, may dissipate following the acute stage of the injury. In our sample, the length of time since lesion (TSL) was not associated with any of the awareness measures. This may explained by the fact that all patients were non-acute cases, tested at least 6 months post-injury. A more detailed study examining the course of deficit awareness from initial injury to post-acute stages may be informative for clinicians attempting to develop rehabilitation programs for individuals with acquired brain injury and deficit awareness. Furthermore, results from the current study showed that left frontal lesion patients showed poorer scores on a number of neurocognitive tasks, including measures of verbal and working memory,and verbal fluency. It is possible therefore that among left prefrontal lesion patients, poor working memory performance may have manifested itself as a failure in goal set maintenance (Duncan, 1993; Braver, Reynolds, \& Donaldson, 2003), which may have contributed to their lowered metacognitive awareness. By contrast, for right prefrontal lesion patients, a potential mechanism to account for awareness impairments is an error monitoring deficit. This may be part of a more general monitoring deficit, as has been proposed to account for cases of persistent anosognosia, where right prefrontal hypoperfusion is also present (Venneri \& Shanks, 2004). Such a deficit may in turn be mediated by their decreased ability to self-sustain a sufficient level of autonomic arousal, as our SCR results would suggest. However, this hypothesis requires further testing among patient groups with persistent anosognosia for hemiplegia or hemiparesis.

On neuropsychological performance, our right prefrontal patients showed no discernable impairment, as evidenced by 
group scaled scores falling well into the average range of performance. This conforms to a widely acknowledged view of right prefrontal lesion patients exhibiting minimal impairment on standard neuropsychological assessment. However, deficit awareness impairment in itself represents a significant challenge for caregivers, family members and clinicians alike. Deficits in error monitoring can be targeted for rehabilitation (see McAvinue, O'Keeffe, McMackin, \& Robertson, 2005), and therefore more systematic research on the factors which give rise to error awareness is required, allowing rehabilitation professionals to make optimal use of findings such as those of the present study.

We acknowledge that our study is limited by the use of small samples of rare clinical populations. Although we grouped patients into unilateral groups according to predominant areas of damage, in order to examine potential hemispheric asymmetry effects, lesions may have additional, more widespread effects on neuronal functioning. Lesions affecting the ventromedial frontal regions for example, are particularly likely to have at least some bilateral effect. Without more detailed investigation of connectivity and functioning of neural pathways, our conclusions necessarily remain somewhat speculative. In addition, while we used a number of questionnaires to measure metacognitive awareness. one methodological drawback here is the use of only one task to assess online emergent awareness. Future studies may wish to assess moment-to-moment error awareness across a number of tasks, to increase validity of this novel approach to assessing awareness.

Overall, our results suggest a strong role of left and right frontal systems for accurate metacognitive and online emergent awareness. No double dissociations between our unilateral patient groups emerged, therefore the specific role of lateralized frontal structures remains uncertain at this point. The current findings should be used as a basis for further exploration of the putative neuroanatomic substrate of self- and error-awareness. Future studies may need to investigate the role of neuropsychological deficits in frontal lobe lesion patients with impaired awareness in more detail, in order to develop neurorehabilitative tools for the mediation of such impairments. Future studies may also wish to conduct more detailed examinations of deficit awareness among patients with more specifically defined nonfrontal lesion patients.

\section{Acknowledgements}

We wish to thank all participants of this study. Our thanks also goes to Paul Dockree and Redmond O'Connell, for valuable support and insightful discussions with the authors, throughout this study.

This research was supported by a Health Research Board (HRB) Ireland grant to D.H.

\section{References}

Abreu, B. C., Seale, G., Scheibel, R. S., Huddleston, N., Zhang. L., \& Ottenbacher, K. J. (2001). Levels of self-awareness after acute brain injury: how patients' and rehabilitation specialists' perceptions compare. Archives of Physical and Medical Rehabilitation, 82, 49-56.

Abu-Akel, A., \& Shamay-Tsoory, S. (2011). Neuroanatomical and neurochemical bases of theory of mind. Neuropsychologia, 49(11), 2971-2984.

Beauregard, M., Levesque, J., \& Bourgouin, P. (2001). Neural correlates of conscious self-regulation of emotion. Journal of Neuroscience, 21, 165RC.

Braver, T. S., Reynolds, J. R., \& Donaldson, D. 1. (2003). Neural mechanisms of transient and sustained cognitive control during task switching. Neuron, 39 , 713-726.

Broadbent, D. E. Cooper, P. F., FitzGerald, P.. \& Parkes, K. R. (1982). The Cognitive Failures Questionnaire (CFQ) and its correlates. British Journal of Clinical Psychology, 21, 1-16.
Crosson, C., Barco, P. P., Velozo, C., Bolesta, M. M., Cooper, P. V., Werts, D., et al. (1989). Awareness and compensation in post-acute head injury rehabilitation. Journal of Head Trauma Rehabilitation, 4, 46-54.

Duncan, J. (1993). Selection of input and goal in the control of behavior. In: A. D. Baddeley, \& L. Weiskrantz (Eds.). Attention: Selection, awareness, and controL A tribute to Donald Broadbent (pp. 53-71). New York: Oxford University Press.

Fleming. J. M., Strong. J., \& Ashton, R. (1996). Self-awareness of deficits in adults with traumatic brain injury: how best to measure? Brain Injury, 10, 1-15.

Grace, J., \& Malloy, P. F. (2002). Frontal systems behavior scale - professional manual. Lutz, Florida: Psychological Assessment Resources.

Jenkinson, P. M., Edelstyn, N. M., Drakeford, J. L., \& Ellis, S. J. (2009). Reality monitoring in anosognosia for hemiplegia. Conciousness and Cognition, $18(2)$ $458-470$

Manly. T., Owen, A. M. McAvinue, L, Datta, A., Lewis, G. H., Scott, S. K., et al. (2003). Enhancing the sensitivity of a sustained attention task to frontal damage: convergent clinical and functional imaging evidence. Neurocase, 9 . 340-349.

McAvinue, L., O'Keeffe, F., McMackin, D., \& Robertson, I. H. (2005). Impaired sustained attention and error awareness in traumatic brain injury: implications for insight. Neuropsychological Rehabilitation, 15, 569-587.

Nelson, H. E. (1982). The National Adult Reading Test (NART): test manual. Windsor: NFER-Nelson.

O'Keeffe, F. M., Dockree, P. M. \& Robertson, 1. H. (2004). Poor insight in traumatic brain injury mediated by impaired error processing? Evidence from electrodermal activity. Brain Research Cognitive Brain Research, 22, 101-112.

O'Keeffe, F. M., Dockree, P. M., Moloney, P., Carton, S., \& Robertson, 1. H. (2007a) Awareness of deficits in traumatic brain injury: a multidimensional approach to assessing metacognitive knowledge and online-awareness. Journal of the Intemational Neuropsychological Society, 13, 38-49.

O'Keeffe, F. M., Murray, B., Coen, R. F., Dockree, P. M., Bellgrove, M. A., Garavan, H. et al. (2007b). Loss of insight in frontotemporal dementia, corticobasal degeneration and progressive supranuclear palsy. Brain, 130, 753-764.

Orfei, M. D., Robinson, R. G., Prigatano, G. P. Starkstein, S., Rusch, N., Bria, P., et al. (2007). Anosognosia for hemiplegia after stroke is a multifaceted phenomenon: a systematic review of the literature. Brain, 130, 3075-3090.

Partington, J. E. \& Leiter, R. G. (1949). Partington's Pathway test. The Psychological Service Centre Bulletin, 1, 9-20.

Pia, L.. Neppi-Modona, M., Ricci, R., \& Berti, A. (2004). The anatomy of anosognosia for hemiplegia: a meta-analysis. Cortex, 40, 367-377.

Prigatano, G. P. (2005). Disturbances of self-awareness and rehabilitation of patients with traumatic brain injury: a 20-year perspective. Journal of Head Trauma Rehabilitation, 20, 19-29.

Prigatano, G. P., Borgaro, S., Baker, J. \& Wethe, J. (2005). Awareness and distress after traumatic brain injury: a relative's perspective. journal of Head Traume Rehabilitation, 20(4), 359-367.

Prigatano, G. P. \& Fordyce, D. J. (1986). Cognitive dysfunction and psychosocial adjustment after brain injury. In: G. P. Prigatano, D. J. Fordyce, H. K. Zeiner, J. R. Rouech, M. Pepping, \& B. Wood (Eds.), Neuropsychological rehabilitation after brain injury (pp. 96-118). Baltimore, ML: John Hopkins University Press.

Robertson, L. H., Manly, T., Andrade, J. Baddeley, B. T., \& Yiend, J. (1997). 'Oops!': performance correlates of everyday attentional failures in traumatic brain injured and normal subjects. Neuropsychologia, 35, 747-758.

Schacter, D. L. \& Prigatano, G. P. (1991) Forms of unawareness, In: D. L. Schacter, \& G. P. Prigatano (Eds.). Awareness of deficit after brain injury: Clinical and theoretical issues (pp. 258-262). New York: Oxford University Press.

Spreen, O., \& Strauss, E. A. (1998). Compendium of neuropsychological tests. Administration, norms and commentary (2nd ed.). New York: Oxford University Press.

Starkstein, S. E. Vazquez, S., Migliorelli, R., Teson, A., Sabe, L. \& Leiguarda, R. 1995). A single-photon emission computed tomographic study of anosognosia in Alzheimer's disease. Archives of Neurology, 52, 415-420.

Stern, R. M. Ray, W. J., \& Quigley, K. S. (2001). Psychophysiological recording. New York: Oxford University Press.

Stuss, D. T. (1991). Disturbance of self-awareness after frontal system damage. In: G. P. Prigatano, \& D. L. Schacter (Eds.), Awareness of deficit after brain injury: clinical and theoretical issues (pp. 63-83). New York: Oxford University Press.

Stuss, D. T., Picton, M. D., \& Alexander, M. P. (2001). Consciousness, self-awareness and the frontal lobes. In: S. P. Salloway, P. F. Malloy, \& J. D. Duffy (Eds.), The frontal lobes and neuropsychiatric illness (pp. 101-112). Washington, D.C. American Psychiatric Press.

Toglia, J. \& Kirk, U. (2000). Understanding awareness deficits following brain injury. NeuroRehabilitation, 15, 57-70.

Venneri, A., \& Shanks, M. F. (2004). Belief and awareness: reflections on a case of persistent anosognosia. Neuropsychologia, 42, 230-238.

Vocat, R., Staub, F, Stroppini, T, \& Vuilleumier, P. (2010). Anosognosia for hemiplegia: a clinical-anatomical prospective study. Brain, 133(12), 3578-3597.

Wechsler, D., Wycherly, R., Benjamin, L., Crawford, J. R., \& Mockler, D. (1998), Wechsier Memory Scale III. London The Psychological Corporation Limited.

Worrall, B. B., Chen, D. T., Dimberg. E. L., \& Katz, J. M. (2005). Should thrombolysis be given to a stroke patient refusing therapy due to profound anosognosia? Neurology, 65, 500

Zahn, T. P., Grafman, J. \& Tranel, D. (1999). Frontal lobe lesions and electroderma) activity: effects of significance. Neuropsychologia, 37, 1227-1241.

Zigmond, A. S., \& Snaith, R. P. (1983). The hospital anxiety and depression scale. Acta Psychiatrica Scandinavia, 67, 361-370. 